\title{
A Case of Laugier-Hunziker Syndrome
}

\author{
Eun Jung Kim, M.D., Sang Hyun Cho, M.D., Jeong Deuk Lee, M.D. \\ Department of Dermatology, Our Lady of Hospital, College of Medicine, \\ The Catholic University of Korea, Incheon, Korea
}

\begin{abstract}
Laugier-Hunziker syndrome is a rare idiopathic acquired hyperpigmentation of oral mucosa and lips which is often associated with longitudinal melanonychia. This condition is known to be an entirely benign disease with no systemic manifestations. Herein we report a case of a 54-year-old woman presenting with a 7-year history of asymptomatic oral pigmentation and linear pigmented streaks of several fingernails. Histological examination taken from the mucosal lesion of tongue revealed increased pigmentation at the epithelial basal laver and a few melanophages in the submucosa. The lack of a family history coupled with the absence of any polyps on the endoscopic examinations of upper and lower bowel as well as the clinical features with late onset were all in favor of a diagnosis of Laugier-Hunziker syndrome. The significance of this disease is due to the need to differentiate this disorder from other pigmentary disorders of the oral mucosa, especially Peutz-Jeghers syndrome.
\end{abstract}

(Ann Dermatol (Seoul) 20(3) 126 129, 2008)

Key Words: Laugier-Hunziker syndrome, Melanonychia, Oral pigmentation

\section{INTRODUCTION}

In 1970, Laugier and Hunziker ${ }^{1}$ described acquired macular hyperpigmentation of the lips and oral mucosa in 5 patients, with longitudinal nail pigmentation in 2 of them. The eponymous terminology "Laugier-Hunziker syndrome" (LHS) was later coined to designate this entity. Additional reports emphasized the absence of associated systemic disease, and the need to differentiate this condition from diseases that have similar pigmentary changes but also significant systemic abnormalities ${ }^{2,3}$. The importance of recognizing this benign syndrome is to avoid unnecessary and invasive diagnostic investigation and treatment. We herein describe a case of a 54-year-old woman with classical findings of Laugier-Hunziker syndrome to underlie the impor-

Received January 11, 2008

Accepted for publication March 4, 2008

Reprint request to: Jeong Deuk Lee, M.D., Department of Dermatology, Our Lady of Mercy Hospital, College of Medicine, The Catholic University of Korea, 665, Bupyeong 6-dong, Bupyeong-gu, Incheon 403-720, Korea. Tel: 82-32510-5528, Fax: 82-32-506-9514, E-mail: leejd@olmh.cuk. ac.kr tance of this differential diagnosis.

\section{CASE REPORT}

A 54-year-old woman presented with a 7-year history of pigmentary change on the lips and intraoral mucosa. She was otherwise healthy with no history of gastrointestinal problems. There was no relevant family history. There was no drug or exposure history to explain the findings. Oral lesions manifested as irregular dark brown to black confluent pigmented macules on the upper and lower lips extending to intraoral surfaces (Fig. 1A). Diffuse pigmentary change with indistinct margins on the lateral and lower aspects of tongue was noted (Fig. 1B). Inspection of her fingernails showed longitudinal hyperpigmented streaks spreading onto the distal nailfolds on several fingers (Fig. 1C). On the physical examination, she was otherwise unremarkable and routine laboratory investigations were all within the normal range. The patient was referred for an opinion by a gastroenterologist. In view of the possibility of Peutz-Jeghers syndrome, she underwent the endoscopic study of upper and lower bowel, 

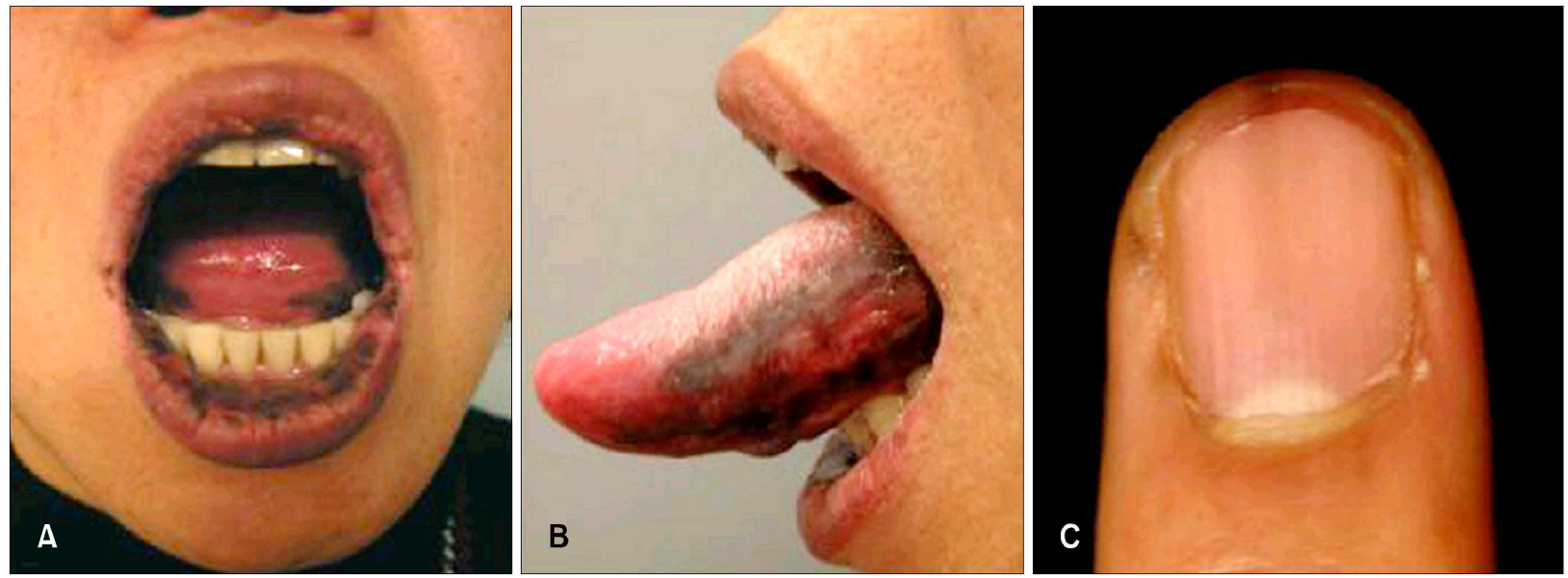

Fig. 1. (A) Dark brown to black, confluent, hyperpigmented macules on the lips and intraoral mucosa. (B) Diffuse hyperpigmentation on the lateral surface of tongue. (C) Longitudinal melanonychia of the right index fingernail. Hyponychium also shows hyperpigmentation.
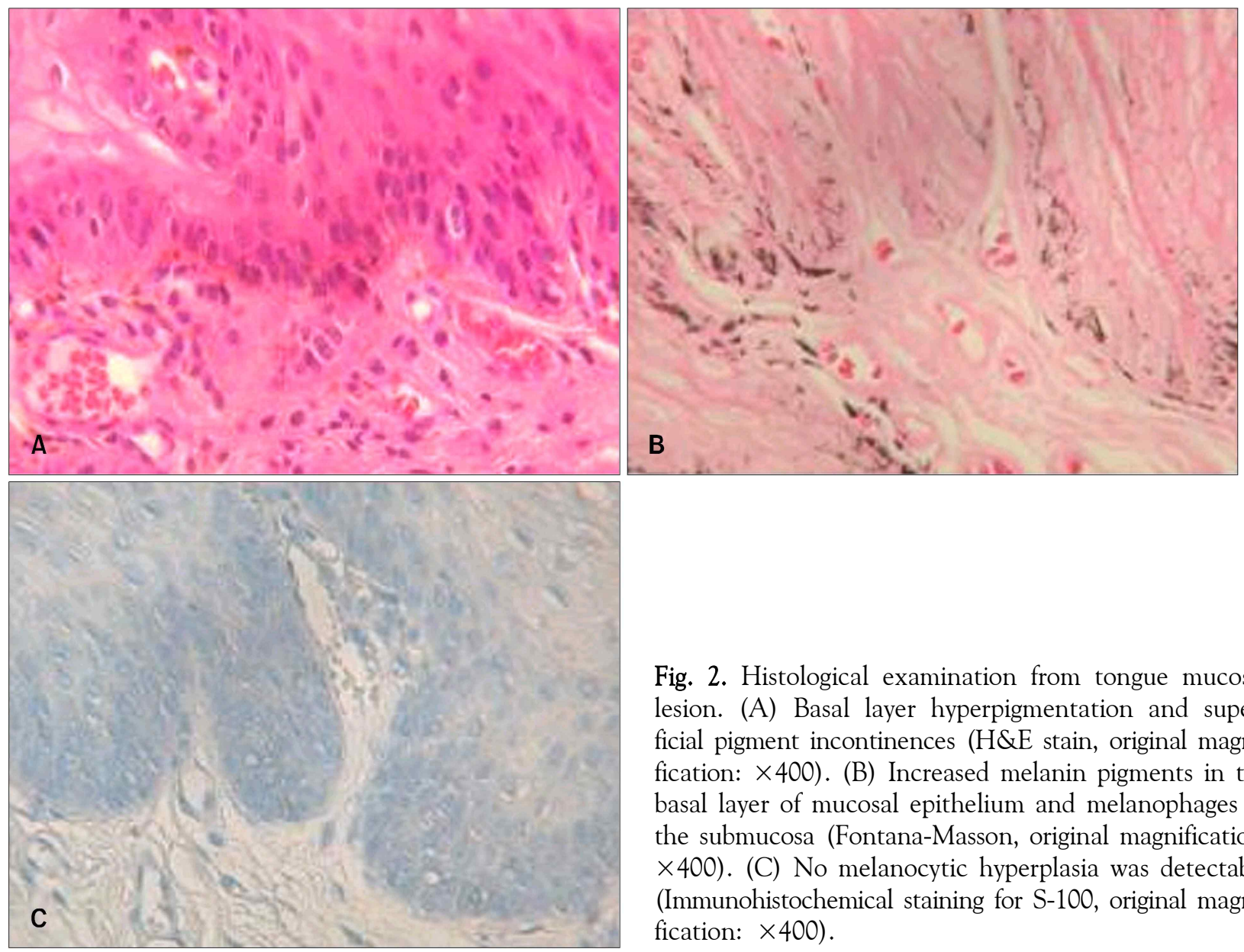

Fig. 2. Histological examination from tongue mucosal lesion. (A) Basal layer hyperpigmentation and superficial pigment incontinences $(H \& E$ stain, original magnification: $\times 400$ ). (B) Increased melanin pigments in the basal layer of mucosal epithelium and melanophages in the submucosa (Fontana-Masson, original magnification: $\times 400$ ). (C) No melanocytic hyperplasia was detectable (Immunohistochemical staining for S-100, original magnification: $\times 400$ ). 
which revealed no evidences of polyps. Histological examination taken from the mucosal lesion of tongue showed increased basal layer hyperpigmentation without melanocytosis and superficial pigmentary incontinence with a few melanophages in the submucosa (Fig. 2). Given the patient's history and findings from clinical and histological examinations, a diagnosis of Laugier-Hunziker syndrome was made. The patient was informed about the entirely benign and sporadic nature of the disease with no systemic involvement. No treatment was desired or administered.

\section{DISCUSSION}

Laugier-Hunziker syndrome is characterized by the presence of a variable number of asymptomatic, discrete, slate to brown-black pigmented macules involving the oral mucosa and lips, which are sometimes confluent or even diffuse ${ }^{3-5}$. Buccal mucosa is commonly affected and reports also include pigmentation of the hard and soft palate, the gingival, the palatoglossal arch, the floor of the mouth and the tongue. Nail changes are found in about 60\% of cases $^{2}$. The characteristic changes are longitudinal pigmented streaks in one or more fingernails ${ }^{6}$. Toenails are less frequently involved than fingernails. Pigmentary change has also been reported on the fingertips and nail folds? ${ }^{7}$. Pigmentation around the proximal nailfold has been termed the pseudoHutchinson's sign and no evidence of malignant changes has been reported ${ }^{8}$. The onset of this condition is usually in middle age and to date there has been no known association with systemic disease. Histologically, pigmentations are due to an accumulation of melanin in the basal layer keratinocytes and an increase in the number of melanophages in the submucosa or papillary dermis. The etiology of this acquired and benign disease remains undetermined.

Several conditions may induce abnormal mucocutanoues and/or nail pigmentation resembling the clinical picture of Laugier-Hunziker syndrome. These include benign racial pigmentation, Addison's disease, Peutz-Jeghers syndrome, LEOPARD syndrome, LAMB syndrome, drug ingestion such as minocycline, antimalarials, and phenothiazines ${ }^{2,3}$. Most of these conditions were ruled out in our patient on the basis of history and clinical examination for the associated features. The Peutz-Jeghers syndrome was considered to be the most important differential diagnosis because the classical occurrence of mucosal and labial pigmentation in this disease may make diagnostic confusion in our patient.

Peutz-Jeghers syndrome is an autosomal dominant disorder characterized by hamartomatous gastrointestinal polyposis and melanin pigmentation of the skin and mucous membranes9. The polyps occur throughout the whole digestive tract with a predilection for the small bowel. Most patients present in adolescence or young adulthood with episodes of colicky abdominal pain caused by recurrent intussusceptions or intestinal obstruction. Pigmentation starts

Table 1. Differentiation between Peutz-Jeghers syndrome and Laugier-Hunziker syndrome

\begin{tabular}{|c|c|c|}
\hline & Peutz-Jeghers syndrome & Laugier-Hunziker syndrome \\
\hline Inheritance & Autosomal dominant & Acquired \\
\hline Age of onset & Infancy or early childhood & Middle aged adult \\
\hline \multicolumn{3}{|l|}{ Mucocutaneous features } \\
\hline Oral pigmentation & Perioral distribution is typically seen & Usually confined to intraoral surface \\
\hline Nail pigmenation & Rare & Frequent (approximately $60 \%$ of cases) \\
\hline Systemic involvement & Hamartomatous gastrointestinal polyposis & None \\
\hline Risk of malignancy & $\begin{array}{l}\text { High risk of gastrointestinal (large bowel, } \\
\text { duodenum, stomach) and extraintestinal } \\
\text { (breast, uterus, cervix, ovaries, testicles, } \\
\text { pancreas) malignancy }\end{array}$ & None \\
\hline Surveillance protocol & $\begin{array}{l}\text { Two yearly entire gastrointestinal investigation, } \\
\text { early breast screening and yearly gynecological } \\
\text { evaluation }\end{array}$ & Unnecessary \\
\hline
\end{tabular}


to appear in infancy or early childhood and occur most commonly on the lips and oral mucosa. In addition, periorificial pigmentation around the mouth, nose and eyes are classically seen, whereas the lesions of the Laugier-Hunziker syndrome are confined to intraoral surfaces. Although pigmentary nail changes have been described in cases of PeutzJeghers syndrome, these are rare $^{10}$. A number of studies have shown a significantly increased risk of malignancy, both gastrointestinal as well as extraintestinal such as the breast, uterus, cervix, ovaries, testicles and pancreas, and the chance of an affected person to die of cancer by the age of 57 has been estimated to be as high as $48 \%{ }^{11}$. Therefore, comprehensive surveillance protocols have been established for patients with Peutz-Jeghers syndrome consisting of two yearly entire gastrointestinal endoscopic investigation, early breast screening, and yearly gynecological evaluation ${ }^{11}$.

Therefore, several pieces of evidence including lack of polyposis, late onset in middle age and no family history of findings typical of Peutz-Jeghers syndrome strongly render this diagnosis unlikely in our patient. Moreover, longitudinal melanonychia together with the absence of periorificial distribution was another piece of evidence in favor of the Laugier-Hunziker syndrome (Table 1). The patient was reassured about the benign and sporadic nature of her condition.

Although there are four reported cases of Korean patients with this condidion ${ }^{12-15}$, we think that this condition is probably more common than is generally recognized, considering that some patients have been mislabeled as Peutz-Jeghers syndrome due to its similarity of mucocutaneous features. In conclusion, Laugier-Hunziker syndrome remains a diagnosis of exclusion. It should be kept in mind when confronted with a patient with pigmentary changes suggestive of Peutz-Jeghers syndrome. Correct clinical identification of this benign syndrome negates the need for unnecessary invasive screening protocols.

\section{REFERNECES}

1. Laugier P, Hunziker H. Pigmentation melaniques lenticulaire de la nuquese jugale et des levres. Arch
Belges Dermatol Syph 1970;26:391-399.

2. Kemmett D, Ellis J, Spencer MJ, Hunter JA. The Laugier-Hunziker syndrome - a clinical review of six cases. Clin Exp Dermatol 1990;15:111-114.

3. Lampe AK, Hampton PJ, Woodford-Richens K, Tomlinson I, Lawrence CM, Douglas FS. LaugierHunziker syndrome: an important differential diagnosis for Peutz-Jeghers syndrome. J Med Genet 2003;40:e77.

4. Mignogna MD, Lo Muzio L, Ruoppo E, Errico M, Amato M, Satriano RA. Oral manifestations of idiopathic lenticular mucocutaneous pigmentation (Laugier-Hunziker syndrome): a clinical, histopathological and ultrastructural review of 12 cases. Oral Dis 1999;5:80-86.

5. Fischer D, Field EA, Welsh S. Laugier-Hunziker syndrome. Clin Exp Dermatol 2004;29:312-313.

6. Sterling GB, Libow LF, Grossman ME. Pigmented nail streaks may indicate Laugier-Hunziker syndrome. Cutis 1988;42:325-326.

7. Sardana K, Mishra D, Garg V. Laugier-Hunziker syndrome. Indian Pediatr 2006;43:998-1000.

8. Lenane P, Sullivan DO, Keane CO, Loughlin SO. The Laugier-Hunziker syndrome. J Eur Acad Dermatol Venereol 2001;15:574-577.

9. Giardiello FM, Trimbath JD. Peutz-Jeghers syndrome and management recommendations. Clin Gastroenterol Hepatol 2006;4:408-415.

10. Velero A, Shert K. Pigmented nails in the PeutzJeghers syndrome. Am J Gastroenterol 1961;42:5658.

11. Hearle N, Schumacher V, Menko FH, Olschwang S, Boardman LA, Gille JJ, et al. Frequency and spectrum of cancers in the Peutz-Jeghers syndrome. Clin Cancer Res 2006;12:3209-3215.

12. Jang KA, Ahn SJ, Choi JH, Sung KJ, Moon KC, Koh JK. Laugier-Hunziker syndrome. Korean J Dermatol 1999;37:108-110.

13. Hong JK, Jung SH, Lee DW, Lee JY, Cho BK. A case of Laugier-Hunziker syndrome. Korean J Dermatol 1999;37:113-115.

14. Pyo WS, Lee JB, Lee SC, Won YH. LaugierHunziker syndrome. Korean J Dermatol 2000;38: 1391-1393.

15. Roh $\mathrm{BH}$, Whang $\mathrm{KU}$, Park $\mathrm{CH}$, Cho MK, Park YL, Lee JS. A case of Laugier-Hunziker syndrome. Korean J Dermatol 2007;45:751-753. 